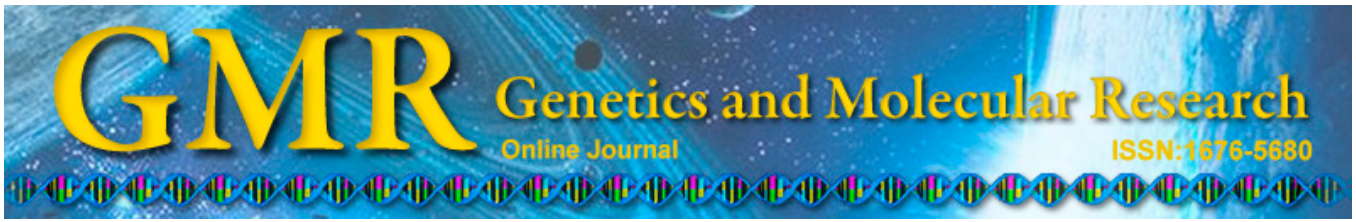

\title{
Development and characterization of genic-SSR markers from different Asia lotus (Nelumbo nucifera) types by RNA-seq
}

X.F. Zheng ${ }^{1 *}$, Y.N. You ${ }^{1 *}$, Y. Diao ${ }^{1,2}$, X.W. Zheng ${ }^{3}$, K.Q. Xie ${ }^{3}$, M.Q. Zhou ${ }^{4}$, Z.L. Hu ${ }^{1,4}$ and Y.W. Wang ${ }^{5}$

${ }^{1}$ State Key Laboratory of Hybrid Rice, College of Life Sciences, Wuhan University, Wuhan, Hubei, China ${ }^{2}$ College of Forestry and Life Sciences, Chongqing University of Arts and Sciences, Yongchuan, Chongqing, China ${ }^{3}$ Guangchang White Lotus Research Institute, Fuzhou, Jiangxi, China ${ }^{4}$ Lotus Center, Wuhan University, Wuhan, Hubei, China

${ }^{5}$ College of Pharmaceutical Sciences, Wuhan University, Hubei, China

*Theses authors contributed equally to this study.

Corresponding authors: Z.L. Hu

E-mail: huzhongli@whu.edu.cn

Genet. Mol. Res. 14 (3): 11171-11184 (2015)

Received January 6, 2015

Accepted May 25, 2015

Published September 22, 2015

DOI http://dx.doi.org/10.4238/2015.September.22.11

ABSTRACT. Nelumbo nucifera is an important economic vegetable and traditional medicine, but available genetic resources remain limited. Next generation sequencing has proven to be a rapid and effective means of identifying genic simple sequence repeat (genic-SSR) markers. This study developed genic-SSRs for $N$. nucifera using Illumina sequencing technology to assess diversity across cultivated and wild lotus. A total of 105,834 uni-contigs were produced with an average read length of 722 bp. Exactly 11,178 genic-SSR loci were identified in 9523 uni-contigs. Di-nucleotide $(64.5 \%)$ was the most abundant SSR, followed by trinucleotide $(23 \%)$, tetra-nucleotide $(8.9 \%)$, penta-nucleotide $(2.5 \%)$, and hexa-nucleotide $(1 \%)$ repeat types. The most common di- and 
tri-nucleotide repeat motifs were AG/CT (51\%) and AAG/CTT (8\%), respectively. Based on these SSRs sequences, 6568 primer pairs were designed, of which 72 primers were randomly selected for synthesis and validation, and 38 in-silico polymorphic primers were obtained using in-house perl scripts. A total of 110 primers were screened in the lotus samples and the results showed that 101 primers yielded amplification products, of which 80 were polymorphs. The number of alleles ranged from 2 to 17 and the PIC (polymorphism information content) ranged from 0.19 to 0.87 with a mean value of 0.55 . An Unweighted Pair Group Method with Arithmetic Mean (UPGMA) dendrogram based on Jaccard's similarity coefficients showed that the correlation between geographical source and genotype was low. This study describes the distribution of genic-SSRs in the expressed portion of the lotus genome. These genic-SSRs have an important role to play in molecular mapping, diversity analysis, and marker-assisted selection strategies in Nelumbo.

Key words: Transcriptome; Genic-SSRs; Nelumbo nucifera; Genetic diversity

\section{INTRODUCTION}

The genus Nelumbo Adans. only contains Nelumbo nucifera and Nelumbo lutea, and has an Asian and North American disjunct distribution pattern. N. nucifera is well known as an economically important ornamental (i.e., flower types) and dietary plant (i.e. rhizome and seed lotus types). In China, it is also used as an important traditional medicine. In the previous study, almost all parts of $N$. nucifera, including the flower, rhizome, and leaf, possessed anti-obesity, anti-inflammatory, anti-pyretic, anti-oxidant, hepatoprotective and free radical scavenging activities (Sohn et al., 2003).

Previous studies on Nelumbo mainly focused on pharmaceutical activities (Liu et al., 2010) and the population genetic diversity of inter simple sequence repeats (ISSR), allozyme, sequence related amplified polymorphism (SRAP), amplified fragment length polymorphism (AFLP), and simple sequence repeat (SSR) markers (Han et al., 2007; Tian et al., 2008b; Hu et al., 2012; Pan et al., 2011; Yang et al., 2012b). Lotus germplasm accessions are not only a useful natural resource, but also an important gene pool. A better evaluation of lotus germplasm genetic diversity is crucial if it is to be utilized in breeding and conservation. Fu et al. (2011) and $\mathrm{Hu}$ et al. (2012) reported genetic variation between $N$. nucifera and N. lutea. Genetic diversity between China and Thailand lotus plants were evaluated using ISSR by Chen et al. (2008) and Li et al. (2010), which improved understanding of lotus germplasm classification. Although the development of a number of SSRs was reported by Tian et al. (2008a), Kubo et al. (2009), Pan et al. (2010), and Xue et al. (2012), few SSR markers have been exploited, particularly in Nelumbo molecular quantity genetic studies and molecular assistance breeding.

SSR makers are randomly repeated 1-6 DNA motifs that are abundant in eukaryote genomes and can mutate rapidly through the loss or gain of repeat units. Thus, microsatellites showing extensive length polymorphism, high polymorphism information content, and co-dominance have been widely used for comparative mapping, DNA fingerprinting, and 
biodiversity studies (Luro et al., 2008). SSR markers are divided into two types: genomicSSRs and genic-SSRs. Genic-SSRs are highly transferable compared to genomic-SSRs, are linked with particular function genes that contribute to phenotype formation, and they can have powerful utility for Marker Assisted Selection (MAS) (Varshney et al., 2005). Technological advances in large-scale RNA-seq should lead to cost-effective, fast, and reliable generation of ESTs (Expression Sequence Tags). The large ESTs are a powerful genetic resource that can be used to detect genic-SSR loci with the bio-information software. Therefore, we performed Nelumbo RNA-seq to develop large numbers of novel and efficient genic-SSR markers.

This paper reports on the generation of a large expressed sequence dataset based on Illumina HiSeq ${ }^{\mathrm{TM}} 2000$ sequencing technology. The frequency and distribution of genic-SSRs from the rhizome and seed transcriptomes were analyzed. A total of 110 primer pairs were selected and synthesized to validate their amplification effect and the relationships among cultivars and wild lotus plants were assessed.

\section{MATERIAL AND METHODS}

\section{Plant materials}

A total of 51 lotus individuals were analyzed in the genetic diversity experiment (Table 1). The group contained 20 cultivated accessions, 29 wild lotus types, and two distance hybrid progeny. The lotus leaves were collected and underwent DNA extraction using the cetrimonium bromide method (CTAB) (Doyle 1987) with a slight modification by adding polyvinylpyrrolidone (PVP) into CTAB extraction buffer (final concentration: 1\%). Genomic DNA quality was confirmed by $0.8 \%$ agarose gel electrophoresis. Apical buds from wild flower lotus (WFL) plants and cultivated rhizome lotus (CRL) plants were harvested, immersed in liquid nitrogen, and stored at $-80^{\circ} \mathrm{C}$ before RNA extraction.

\section{Construction and solexa sequencing of Nelumbo cDNA libraries}

Total RNA was isolated from WFL and CRL apical buds using TRIzol (Invitrogen, Carlsbad, CA, USA) following the manufacturer protocols and the total mRNA was then purified using a Micropoly (A) Purist ${ }^{\mathrm{TM}}$ mRNA purification kit (Ambion, USA). The cDNAs were synthesized following the modified method reported by $\mathrm{Ng}$ et al. (2005) and then shattered into fragments that were 300 to $500 \mathrm{bp}$ long. After purifying them using Ampure beads (Agencourt, Beverly, MA, USA), cDNA libraries were constructed with TruSeq ${ }^{\mathrm{TM}}$ DNA Sample Prep Kit Set A (Illumina, USA). After amplification using a TruSeq PE Cluster Kit (Illumina), the products were sequenced on an Illumina sequencing platform (Illumina Inc. San Diego, CA, USA) using a 100-bp paired-end approach.

\section{Genic-SSR identification, primer design, and PCR amplification}

A perl program called MISA (Thiel et al., 2003) was used to identify genic-SSR loci from the WFL, CRL, and their combined sequence databases. The search parameters were di-, tri-, tetra-, penta-, and hexa-nucleotide motifs with minimum repeat numbers of $6,5,4,4$, and 4 , respectively. 
Table 1. Information about the lotus samples used in this study.

\begin{tabular}{|c|c|c|c|c|c|}
\hline No. & Name & Species or origin & Sample & Source & Type \\
\hline 1 & Thailand lotus-I & Nelumbo nucifera & Seed lotus & Thailand & Wild \\
\hline 2 & Thailand lotus-II & N. nucifera & Seed lotus & Thailand & Wild \\
\hline 3 & Thailand lotus-III & N. nucifera & Seed lotus & Thailand & Wild \\
\hline 4 & Thailand lotus-IV & N.nucifera & Seed lotus & Thailand & Wild \\
\hline 5 & Thailand lotus- $\mathrm{V}$ & N. nucifera & Seed lotus & Thailand & Wild \\
\hline 6 & Thailand lotus-VI & N. nucifera & Seed lotus & Thailand & Wild \\
\hline 7 & Cunsan lotus & N. nucifera & Seed lotus & Xiangtan, Hunan & Local Variety \\
\hline 8 & Furong lotus & N. nucifera & Seed lotus & Xiangtan, Hunan & Local Variety \\
\hline 9 & Baixiang lotus & N. nucifera & Seed lotus & Xiangtan, Hunan & Local Variety \\
\hline 10 & Hongxiagn lotus & N. nucifera & Seed lotus & Xiangtan, Hunan & Local Variety \\
\hline 11 & Yueyang wild lotus & N. nucifera & Seed lotus & Yueyang, Hunan & Wild \\
\hline 12 & Xuehugong lotus & N. nucifera & Rhizome lotus & Anqing, Anhui & Wild \\
\hline 13 & Nangeng wild lotus & N. nucifera & Rhizome lotus & Ma'anshan, Anhui & Wild \\
\hline 14 & Piaohua lotus & N. nucifera & Rhizome lotus & Anhui & Wild \\
\hline 15 & Lianhu wild lotus & N. nucifera & Rhizome lotus & Anhui & Wild \\
\hline 16 & Madang lotus & N. nucifera & Rhizome lotus & Anhui & Wild \\
\hline 17 & Shanmiao wild lotus & N. nucifera & Rhizome lotus & Anhui & Wild \\
\hline 18 & Qingtang wild lotus & N. nucifera & Rhizome lotus & Anhui & Wild \\
\hline 19 & Baihu wild lotus & N. nucifera & Rhizome lotus & Anhui & Wild \\
\hline 20 & Chuzhoubai lotus & N. nucifera & Seed lotus & Jinhua, Zhejiang & Local Variety \\
\hline 21 & Tuxuan lotus & N. nucifera & Seed lotus & Jinhua, Zhejiang & Local Variety \\
\hline 22 & Baihuajian lotus & N. nucifera & Seed lotus & Jianning, Fujian & Local Variety \\
\hline 23 & Honghuajian lotus & N. nucifera & Seed lotus & Jianning, Fujian & Local Variety \\
\hline 24 & Heilongjiang wild lotus & N. nucifera & Seed lotus & Beijing & Wild \\
\hline 25 & Dajinhu lotus & N. nucifera & Seed lotus & Beijing & Wild \\
\hline 26 & Zhaoyuan wild lotus & N. nucifera & Seed lotus & Beijing & Wild \\
\hline 27 & Guangchangbaiye lotus & N. nucifera & Seed lotus & Guangchang, Jiangxi & Local Variety \\
\hline 28 & Jingguang-2 hao & N. nucifera & Seed lotus & Guangchang, Jiangxi & Local Variety \\
\hline 29 & Xingkongmudan & N. nucifera & Seed lotus & Guangchang, Jiangxi & Local Variety \\
\hline 30 & Gudai lotus & N. nucifera & Seed lotus & Wuhan, Hubei & Wild \\
\hline 31 & Zhongnanhaigu lotus & N. nucifera & Seed lotus & Wuhan, Hubei & Wild \\
\hline 32 & Puzheheibai lotus & N. nucifera & Seed lotus & Wuhan, Hubei & Wild \\
\hline 33 & Wufei lotus & Hybrid & Flower louts & Beijing & Local Variety \\
\hline 34 & Baiyangdianhong lotus & N. nucifera & Flower lotus & Nanjing, Jiangsu & Wild \\
\hline 35 & Zhuanshang lotus & N. nucifera & Flower louts & Beijing & Local Variety \\
\hline 36 & Jiandehonghua lotus & N. nucifera & Flower louts & Jiande, Zhejiang & Local Variety \\
\hline 37 & Yixian lotus & Hybrid & Flower louts & Nanjing, Jiangsu & Local Variety \\
\hline 38 & Qiushuichagntian & N. nucifera & Flower louts & Guangchang, Jiangxi & Local Variety \\
\hline 39 & Chongtai lotus & N. nucifera & Flower louts & Wuhan, Hubei & Local Variety \\
\hline 40 & Donggua lotus & N. nucifera & Flower louts & Changsha, Hunan & Local Variety \\
\hline 41 & Taikong 36 hao & N. nucifera & Seed lotus & Guangchang, Jiangxi & Local Variety \\
\hline 42 & American lotus-I & N. lutea & Flower louts & America & Wild \\
\hline 43 & American lotus-II & N. lutea & Flower louts & America & Wild \\
\hline 44 & American lotus-III & N. lutea & Flower louts & America & Wild \\
\hline 45 & American lotus-IV & N. lutea & Flower louts & America & Wild \\
\hline 46 & American lotus-V & N. lutea & Flower louts & America & Wild \\
\hline 47 & American lotus-VI & N. lutea & Flower louts & America & Wild \\
\hline 48 & Riza 3 hao-I & N. nucifera & Seed lotus & Wuhan, Hubei & Local Variety \\
\hline 49 & Riza 3 hao-II & N. nucifera & Seed lotus & Wuhan, Hubei & Local Variety \\
\hline 50 & WFL* & N. nucifera & Seed lotus & Diaocha Lake, Hubei & Wild \\
\hline 51 & CRL* & N.nucifera & Rhizome lotus & Wuhan, Hubei & Local Variety \\
\hline
\end{tabular}

*Sample was used for Illumina transcriptome sequencing.

The genic-SSR primers were designed using Primer3.0 (Rozen and Skaletsky, 2000) and its default parameters, and the combined sequence database. We selected 72 primers to validate the SSR markers and screened 51 lotus samples. PCR amplification was performed using gradient PCR analysis with annealing temperatures between $54^{\circ}$ and $65^{\circ} \mathrm{C}$ in a $15 \mu \mathrm{L}$ reaction volume containing $1 \mathrm{X}$ buffer, $1.4 \mathrm{mM} \mathrm{MgCl}, 0.1 \mathrm{mM}$ dNTPs, 10 pmol of each primer, $0.5 \mathrm{U}$ Taq polymerase, and $\sim 50 \mathrm{ng}$ template DNA. The PCR products were detected as described by Han et al. (2008). 


\section{In-silico analysis of genic-SSR polymorphism in lotus flowers and rhizomes}

MISA tools was used to locate the position of the SSR loci in the WFL, CRL, and their combined sequence databases. Perl scripts were then compiled and used to search for common and polymorphic genic-SSR markers between WFL and CRL (Figure 1). Firstly, genic-SSR loci from WFL and CRL were identified, and if the forward and rear 15-bp nucleotides beside the SSR locus from WFL and CRL were identical, these SSRs were considered as potential polymorphic loci; secondly, the in-silico polymorphic genic-SSRs were confirmed based on the different number of SSR locus repeats; and thirdly, the polymorphic primers were retrieved from a primers database designed using Primer3.0 (Rozen and Skaletsky, 2000) and the combined sequence database. Using this method, 1627 common genic-SSRs loci were found between WFL and CRL, of which only 48 loci were polymorphic and could be used to search the primer databases. Finally, 38 polymorphic primers were identified and synthesized for validation by PCR amplification and electrophonic detection as described above.

\section{Data analysis}

The raw reads were first filtered to remove low quality reads $(<$ Q20) and all adaptor sequences, and then assembled with the Trinity software into contigs (Grabherr et al., 2011). Finally, three database sets for N. nucifera were constructed (Figure 1).

To estimate the allelic variation for genic-SSRs in the 51 samples, the PIC (Polymorphism Information Content) of each primer was calculated based on the following formula:

$$
\mathrm{PIC}=1-\sum_{\mathrm{i}=0}^{\mathrm{n}} \mathrm{Pi}^{2}
$$

where $\mathrm{Pi}$ is the frequency of the $\mathrm{i}^{\text {th }}$ allele and $\mathrm{n}$ is the total number of alleles amplified for a given genic-SSR marker. The coefficient of genetic similarity among all samples was calculated using NTSYS-pc Version 2.10 (Rohlf, 2000). A dendrogram was constructed based on the genetic similarity matrix using the UPGMA algorithm.

\section{RESULTS}

\section{Assembly of raw reads by Illumina sequencing}

A total of 50,578,940 and 49,452,590 filtered sequence reads were obtained from WFL and CRL, respectively (Figure 1). The total length of the reads was over $9 \mathrm{Gbp}$. The two sets of reads data were subsequently de novo assembled by the Trinity software (Grabherr et al., 2011) into 111,925 and 100,016 contigs, respectively, with lengths of over $200 \mathrm{bp}$, and then further assembled into 105,834 uni-contigs with an average length of $722 \mathrm{bp}$. The size distribution of these contigs is shown in Figure 2. 


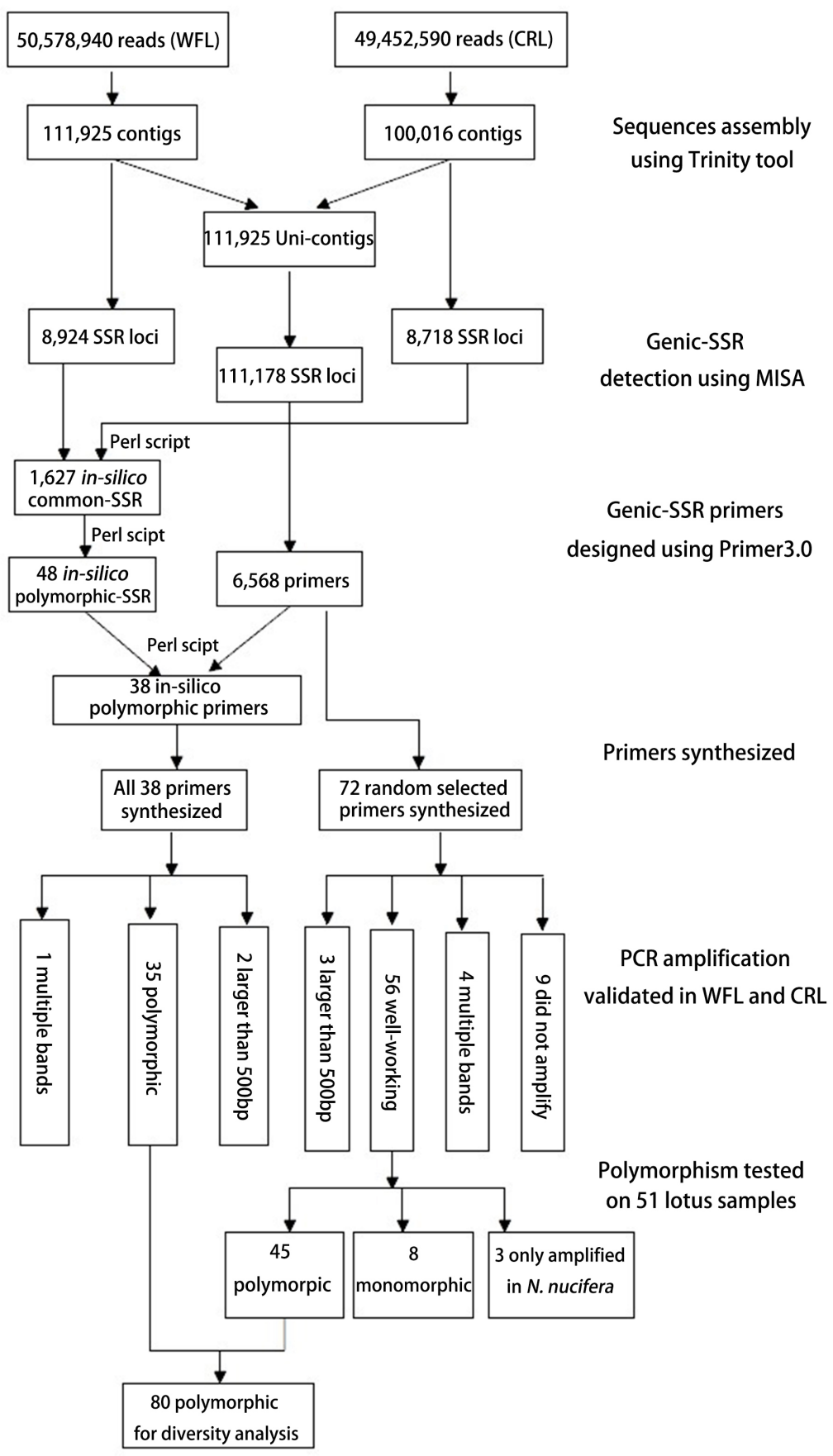

Figure 1. Flow chart of genic-SSR development and diversity analysis from WFL, CRL, and Uni-contigs, respectively. 


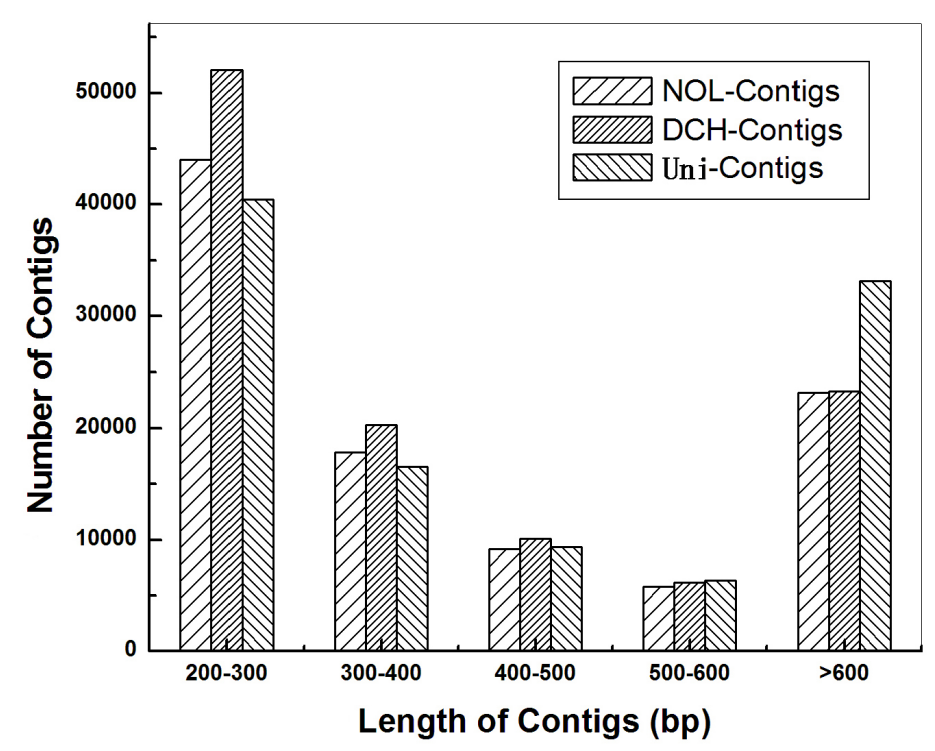

Figure 2. Size distribution of contigs from WFL, CRL, and combined sequences respectively.

\section{Identification, frequency, and distribution of different types of genic-SSR loci}

We used MISA tools to detect 11,178 genic-SSRs loci from 9523 of 105,834 uni-contigs, of which 1345 contained more than one SSR. The distribution density was one genic-SSR locus per $6.84 \mathrm{~kb}$. Among the identified SSR repeats, di-nucleotide (7214, 64.5\%) was the most abundant repeat unit, followed by tri- $(2576,23 \%)$, tetra- $(998,8.9 \%)$, penta- $(279,2.5 \%)$, and hexa- $(111,1 \%)$ nucleotides (Figure 3a). Clearly, there were large proportions of di-nucleotide and tri-nucleotide motifs, whereas the remainder made up less than $12.5 \%$ in total. The average frequency of genic-SSR occurrence was about $9 \%$. The number of SSR repeats ranged from 4 to 31, and SSRs with six repeats $(2704,24.2 \%)$ were the most abundant (Figure 3b), followed by those with seven tandem repeats $(1941,17.4 \%)$, five tandem repeats $(1696,15.2 \%)$, and eight tandem repeats $(1535,13.7 \%)$. Single sample repeat loci containing $12 \mathrm{bp}$ were the most common (1962, 17.6\%) followed by those with 14 bp (2247, 20.1\%), 18 bp (2018, 18.1\%), 14 bp (1564, $14 \%)$, and $15 \mathrm{bp}(1454,13 \%)$ (Figure 3c). The longest SSR locus was $102 \mathrm{bp}$.

A total of 182 repeat motif types were identified, based on sequence complementary. The AG/CT repeat motif $(5714,51.1 \%)$ was the most common. The six next most abundant repeat motifs were (AAG/CTT)n, (AC/GT)n, (AT/AT)n, (ATC/ATG)n, (AAAT/ATTT)n, and (AGG/CCT)n, with frequencies of $8.2,7.1,6.2,4,3$, and $2 \%$, respectively. The most common 12 repeat motifs are shown in Table 2.

\section{Development and validation of genic-SSRs markers}

Primer3.0 (Rozen and Skaletsky, 2000) and the non-redundant uni-contig sequences, which contained 11,178 genic-SSR loci in total, were used to design 6568 primer pairs (Table S1), and 72 primer pairs were synthesized to validate their amplification effect (Table S2). Among these primer pairs, PCR successfully amplified $63(87.5 \%)$ primer pairs using 

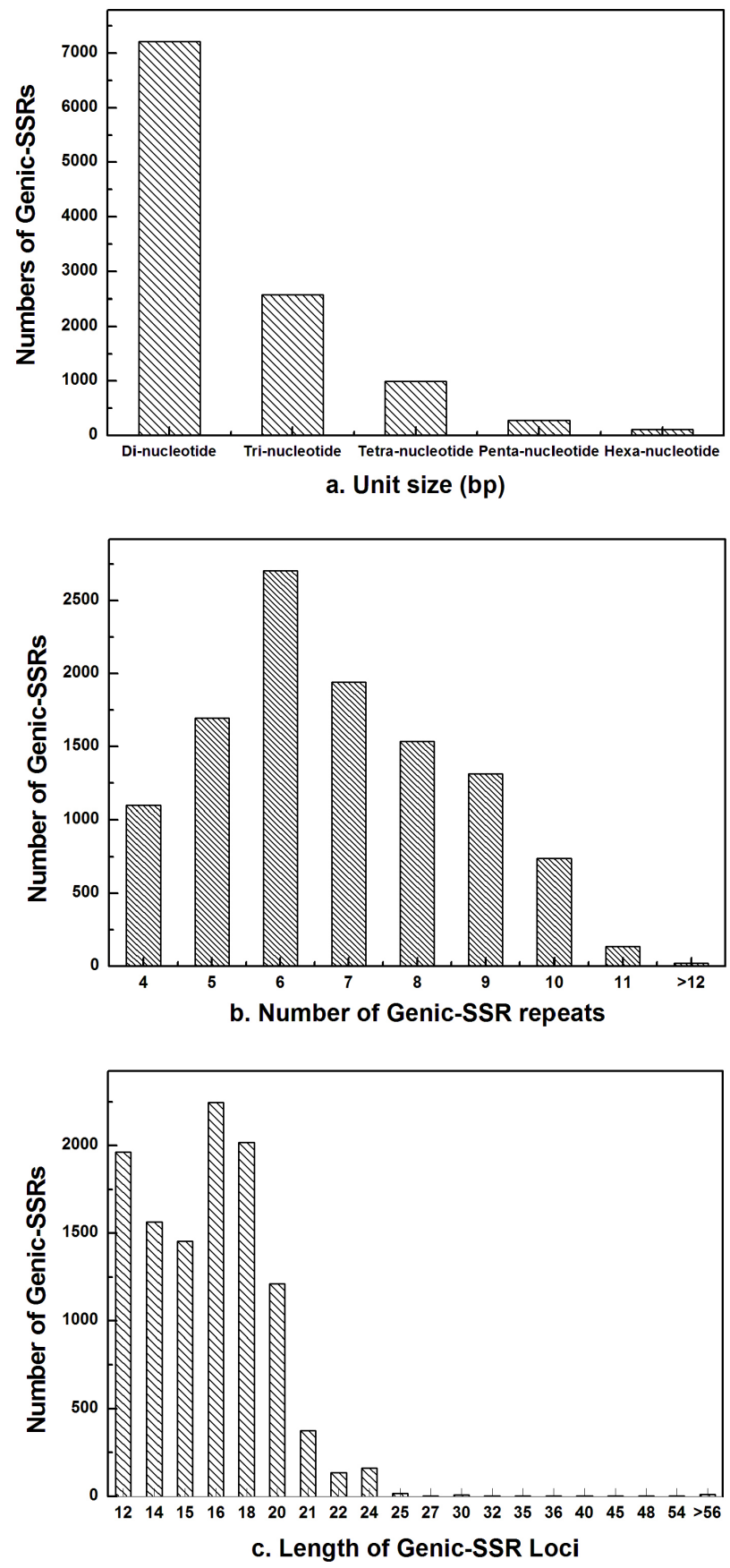

Figure 3. Frequency distribution of the genic-SSRs of differents sizes from the lotus uni-contigs. a. Unit size: genic-SSR motif length. b. Number of genic-SSR repeat: the number of genic-SSR repeat unit. c. Length of genicSSR loci the length of genic-SSR repeat unit. 
Table 2. Frequency distribution of the 12 most frequent genic-SSR repeat motifs in the lotus transcriptome and the number of repeats within each motif.

\begin{tabular}{rlrrrrrrrrrrrr}
\hline Order & Repeats & 4 & 5 & 6 & 7 & 8 & 9 & 10 & 11 & 12 & $>13$ & Total & $\%$ \\
\hline 1 & AG/CT & - & - & 1417 & 1219 & 1215 & 1153 & 618 & 89 & 3 & - & 5714 & $51 \%$ \\
2 & AAG/CTT & - & 462 & 295 & 161 & 2 & - & - & - & - & 1 & 921 & $8 \%$ \\
3 & AC/GT & - & - & 309 & 187 & 137 & 75 & 60 & 25 & 2 & - & 795 & $7 \%$ \\
4 & AT/AT & - & - & 232 & 156 & 148 & 80 & 59 & 21 & 1 & 1 & 698 & $6 \%$ \\
5 & ATC/ATG & - & 261 & 127 & 60 & 3 & - & - & - & - & 1 & 452 & $4 \%$ \\
6 & AAAT/ATTT & 287 & 60 & 5 & - & - & - & - & - & - & - & 352 & $3 \%$ \\
7 & AGG/CCT & - & 137 & 64 & 25 & 4 & - & - & - & - & - & 230 & $2 \%$ \\
8 & AAT/ATT & - & 118 & 57 & 50 & 3 & - & - & - & - & 1 & 229 & $2 \%$ \\
9 & ACC/GGT & - & 144 & 48 & 29 & 4 & 1 & - & - & - & - & 226 & $2 \%$ \\
10 & AGC/CTG & - & 150 & 47 & 18 & 2 & - & - & - & 1 & - & 218 & $2 \%$ \\
11 & AAAG/CTTT & 135 & 55 & 7 & - & - & - & - & - & - & - & 197 & $2 \%$ \\
12 & AAC/GTT & - & 96 & 37 & 18 & - & 1 & - & - & - & - & 152 & $1 \%$ \\
13 & Others & 675 & 213 & 59 & 18 & 17 & 2 & 1 & - & 1 & 8 & 994 & $9 \%$ \\
& Total & 1097 & 1696 & 2704 & 1941 & 1535 & 1312 & 738 & 135 & 8 & 12 & 11,178 & $100 \%$ \\
\hline
\end{tabular}

genomic DNA from WFL and CRL, of which four (5.6\%) amplified many bands with nonspecific products, and the remaining 9-pair primers (12.5\%) produced no products, even when the annealing temperature was reduced by $7{ }^{\circ} \mathrm{C}$. A total of 56 functioning primer pairs, not including three primers that amplified PCR products more than $500 \mathrm{bp}$ long, were screened in the 51 samples, of which $45(80.4 \%)$ showed polymorphisms and three primers $(5.4 \%)$ only amplified PCR bands in N. nucifera. The other eight primers (14.3\%) did not show polymorphism. A total of 189 alleles were identified across 45 polymorphic genic-SSR loci, and the number of alleles ranged from 2 to 10 with an average of 4.2 alleles per locus. In addition, two primers amplified fragments that were larger than the expected sizes, probably because of the presence of an insertion mutation. Only one primer pair amplified a shorter than expected fragment, suggesting that a deletion mutation had occurred in the amplified region.

In order to evaluate and characterize these polymorphisms so that they can be potentially used for assessing molecular diversity or fingerprinting analysis, the PIC values of these polymorphism primers were calculated, based on the allelic variation shown in the 51 lotus accessions. The PIC values across 45 loci ranged from 0.19 for NL-60 to 0.87 for NL-7 with a mean value of 0.52 . At the same time, the likely functions of these genic-SSR loci were deduced by BLAST analysis, and this showed that 16 transcriptomic sequences shared clear homology to other functional loci in plants ( $\underline{\text { Table } \mathbf{S 3}}$ ).

\section{In-silico analysis of genic-SSRs polymorphism between WFL and CRL}

In order to identify highly polymorphic genic-SSR markers between WFL and CRL, in-silico polymorphism analysis of genic-SSRs was undertaken using in-house perl scripts. Contig databases were obtained from WFL and CRL using Trinity tool, and positional information for the genic-SSRs was found using MISA. The in-house perl scripts showed that 1627 SSR loci were common between WFL and CRL, of which only 48 SSR loci were polymorphic. Furthermore, 38 of these in-silico polymorphic SSRs could be used to design primers. Therefore, these primers, named NL-P1 to NL-P38, were synthesized and validated in 51 lotus samples (Table S2). The results showed that the NL-P28 primer produced multiple bands, but no specific product, and the size amplification of two primers (NL-P11 and NLP19) were larger than $500 \mathrm{bp}$ in size. The results for the other 35 primers showed that all 35 
primers could successfully amplify polymorphic bands. Finally, 198 alleles were produced from 35 loci, which was consistent with the in-silico analysis results. The number of alleles ranged from 2 to 17 with an average of 5.66 alleles per locus. Across these 35 loci, PIC values ranged from 0.19 for NL-P16 and NL-P27 to 0.87 for NL-P4 with a mean value of 0.57 . The amplification details of these polymorphic primers were listed in $\underline{\text { Table S3 }}$.

\section{Assessment of genetic diversity among cultivated and wild lotus accessions}

The genetic diversity of different varieties and wild lotus were analyzed using 80 polymorphic primers. An UPGMA dendrogram based on Jaccard's similarity coefficients was constructed with three distinct clusters at a cut-off similarity index of 0.64 (Figure 4). The genetic similarity among the 51 lotus types ranged from 0.48 to 1.00 . Cluster I consisted all $N$. nucifera accessions, which was divided into two sub-clusters with a similarity coefficient of 0.69. Sub-cluster Ia included eight seed lotus accessions, nine rhizome lotus accessions and one flower lotus accession. Sub-cluster Ib consisted of 19 seed lotus, five flower lotus and one rhizome lotus accession. Cluster II was composed of two hybrid lotuses that were produced by hybridization between $N$. nucifera and $N$. lutea. All six American lotus accessions that belonged to $N$. lutea were grouped into Cluster III.

In addition, the six lotus accessions from Thailand were divided into two sub-clusters containing lotus accessions from China and lotus accessions from different provinces in China were grouped together. Wild lotus and local cultivated varieties were also grouped into one cluster. This clustering was based on their appearance and utilization value. In the contrast, the geographic sources of the samples were not consistent with the genetic distances among lotus individuals. These results were consistent with previous studies (Pan et al., 2010, 2011; Yang et al., 2012b).

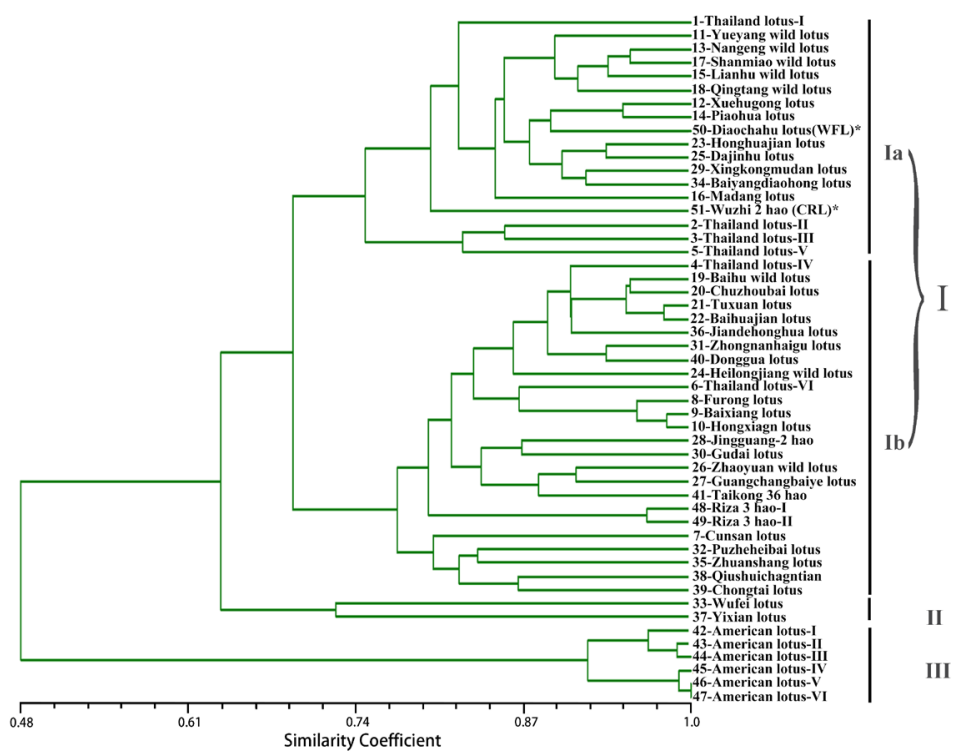

Figure 4. An UPGMA dendrogram of 51 lotus samples based on 80 polymorphic genic-SSR markers. Dendrogram showing similary relationship among 20 cultivated accessions, 29 wild lotus and 2 hybrid progeny, the number 1-51 corresponded to the samples listed in Table 1. 


\section{DISCUSSION}

\section{Frequency and distribution of different types of genic-SSRs loci}

Genic-SSRs have considerable genetic analysis and linkage map construction potential in plants due to their high transferability and conservation. After Pan et al. (2010) reported the development of 23 EST-SSR markers using Nelumbo EST sequences in 2010, there have been no more reports of EST sequences being used for EST-SSRs development in the NCBI database. This study identified a total of 11,178 genic-SSR loci based on 105,834 nonredundant contigs. About $9 \%$ (9523 contigs) of the transcriptomic sequences possessed SSR loci. This rate was higher than for Epimedium sagittatum (3.67\%), which were identified using the same search parameters (Zeng et al., 2010). The distribution density in N. nucifera is one microsatellite loci per $6.84 \mathrm{~kb}$. This Nelumbo genic-SSR frequency occurrence is relatively low compared to some plants, such as $3.4 \mathrm{~kb}$ in rice and $5.4 \mathrm{~kb}$ in wheat, but higher than the 7.4 $\mathrm{kb}$ in soybean, $14 \mathrm{~kb}$ in Arabidopsis, and $20 \mathrm{~kb}$ in cotton (Peng and Lapitan, 2005). Interesting, the SSR frequency (9.33\%) (Yang et al., 2012a) in the Nelumbo genome sequences was lower than the SSR frequency in the transcriptomic sequences. Furthermore, SSR frequency is affected by many factors, such as the search parameters for exploring microsatellite markers, the determination tools, and species type.

In this study, the di-nucleotide repeat was most common in the $N$. nucifera transcriptome, just as in many crops, including Rosaceae (Dutta et al., 2011), sesame (Jung et al., 2005), Pinus contorta (Parchman et al., 2010), sweet potato (Wang et al., 2010), and pigeonpea (Jung et al., 2005; Wei et al., 2008; Parchman et al., 2010; Wang et al., 2010; Dutta et al., 2011). A total of 182 genic-SSR motif types were detected, of which the most dominant di- and tri-nucleotide repeat motifs were AG/CT (51\%) and AAG/CTT (8\%), respectively. The same motif proportions have been observed in many plants, e.g. pigeonpea (Wang et al., 2010; Dutta et al., 2011). Interestingly, the CCG/CGG and CG/CG motifs have the lowest dominant repeat type in $N$. nucifera, which is consistent with the results from dicotyledonous plants (Kumpatla and Mukhopadhyay 2005), such as Epimedium sagittatum (Jiang et al., 2012) and radish (Zeng et al., 2010; Jiang et al., 2012). These results showed that the CCG/ CGG motif is the rarest motif in a large number of dicotyledonous plants. Interestingly the CCG/CGG motif proportion was high in monocots. This might be due to the high GC content and consequent codon usage bias in monocots (Morgante et al., 2002; La Rota et al., 2005). Moreover, the sequences containing CCG/CGG repeats might form potential higher structure, such as hairpins, and thus influence the efficiency and accuracy of RNA splicing (Coleman and Roesser, 1998; Zeng et al., 2010).

\section{Development and validation of genic-SSRs markers}

We synthesized and validated 110 primer pairs in order to evaluate the level of polymorphism so that new genic-SSR markers can be developed. A total of 101 primer pairs (91.8\%) successfully yielded PCR products, which was in line with previous reports that generally found that $60-92 \%$ of primer pairs would be successfully amplified (Xin et al., 2005; Cloutier et al., 2009; Zhang et al., 2012). A total of 72 primers were randomly selected and synthesized, of which, 56 functioning primers were identified in 51 samples. The number of polymorphic primers was $45(80.4 \%)$. This ratio was consistent with that for EST-SSRs in 
other plants, which range from 40 to $89 \%$ (Zhang et al., 2012). Genic-SSRs are generally thought to be less polymorphic than genomic-SSRs. This study supported this, and the polymorphism percentage (80.4\%) was consistent with the $87 \%$ reported by Pan et al. (2010) for lotus. The in-silico polymorphism analysis between WFL and CRL found 1627 common genic-SSRs, of which $48(3 \%)$ possessed polymorphic features. This indicated that there was a close relationship between WFL and CRL, which was supported by the dendrogram based on SSR genotyping data. However, seven polymorphic primers (9.7\%, data not shown) were detected in both WFL and CRL from 72 randomly selected primers. This percentage is obviously higher than those obtained from the in-silico polymorphism analysis (3\%). The differences between the two percentages may be caused by sequencing biases, in that high conserved sequences may be easier to detect simultaneously in WFL and CRL due to their relatively high abundance.

A total of 387 alleles were detected of 80 polymorphic primer pairs. These ranged from 2 to 17 per locus, which is higher than for the N. nucifera genomic-SSRs, which ranged from 2 to 5 per locus according to Kubo et al. (2009) and from 2 to 7 according to Tian et al. (2008a). The average number of alleles from the 80 loci (4.8 alleles) was also higher than the 3.88 (Tian et al., 2008a) and 3.9 alleles (Kubo et al., 2009) reported for genomic-SSR loci. The PIC values for each SSR locus ranged from 0.19 to 0.87 with a mean value of 0.55 . The informativeness value for these EST-SSR markers (0.55) was a little higher than the genomic SSR marker value (0.51) for N. nucifera reported by Kubo et al. (2009); Pan et al. (2010) also reported 23 genic-SSRs from EST sequences found in public databases. The PIC values ranged from 0.02 to 0.61 with a mean of $0.33 \pm 0.17$, and the number of alleles per locus ranged from two to five, with an average of 2.65 alleles. The genic-SSRs obtained in this study have large PIC values and a considerable number of alleles. A preliminary linkage map of Nelumbo using a pseudo-testcross strategy has been constructed by Yang et al. (2012a). The development of these genic-SSRs will strongly support quantitative genetics research and molecular assisted selection, especially the functional gene mapping of lotus.

\section{Assessment of genetic diversity among varieties and wild lotus}

An UPGMA dendrogram was constructed based on genetic similarity results for 80 polymorphic genic-SSR markers. A total of 51 lotus plants were divided into three clear groups. $N$. nucifera (Cluster I) and $N$. lutea (Cluster III) were sub-clustered into two genetically distinct groups (Figure 4). All N. nucifera accessions were divided into two sub-clusters (Clusters Ia and Ib) based on their appearance and utilization value. The same results were reported by Yang et al. (2012b) and Li et al. (2010). Interestingly, the genetic relationship between these lotus accessions was not based on their geographical sources, as was found in previous reports (Guo et al., 2007). Currently, lotuses were classified into three types based on their morphological features and usage values during breeding (Nguyen, 2001; Guo et al., 2007; Guo, 2009). The cluster results showed that there was considerable genetic differentiation between the lotus rhizome (mainly Cluster Ia) and flower (mainly Cluster Ib), except for two accessions (19-Baihu wild lotus and 34-Baiyangdianhong). Wild lotuses have similar clustering to local varieties, which indicated that these local lotus varieties may be selected from wild lotus and gradually formed into cultivated resources without the use of systemic breeding technology. In summary, the relationships among lotuses gained in this diversity evaluation study will improve future breeding research efforts using cultivated varieties and wild lotus resources. 


\section{ACKNOWLEDGMENTS}

We thank Chen Dong for help in improving the English grammar. Research supported by a grant from the National Science and Technology Supporting Program (\#2012BAD27B01).

\section{Supplementary material}

\section{REFERENCES}

Chen YY, Zhou RC, Lin XD, Wu KQ, et al. (2008). ISSR analysis of genetic diversity in sacred lotus cultivars. Aquat. Bot. 89: 311-316.

Cloutier S, Niu Z, Datla R and Duguid S (2009). Development and analysis of EST-SSRs for flax (Linum usitatissimum L.). Theor. Appl. Genet. 119: 53-63.

Coleman TP and Roesser JR (1998). RNA secondary structure: an important cis-element in rat calcitonin/CGRP premessenger RNA splicing. Biochemistry 37: 15941-15950.

Doyle JJ (1987). A rapid DNA isolation procedure for small quantities of fresh leaf tissue. Phytochem. Bull. 19: 11-15.

Dutta S, Kumawat G, Singh BP, Gupta DK, et al. (2011). Development of genic-SSR markers by deep transcriptome sequencing in pigeonpea [Cajanus Cajan (L.) Millspaugh]. BMC Plant Biol. 11: 17.

Fu J, Xiang QY, Zeng XB, Yang M, et al. (2011). Assessment of the genetic diversity and population structure of lotus cultivars grown in China by amplified fragment length polymorphism. J. Am. Soc. Hortic. Sci. 136: 339-349.

Grabherr MG, Haas BJ, Yassour M, Levin JZ, et al. (2011). Full-length transcriptome assembly from RNA-Seq data without a reference genome. Nat. Biotechnol. 29: 644-652.

Guo HB (2009). Cultivation of lotus (Nelumbo nucifera Gaertn. ssp nucifera) and its utilization in China. Genet. Resour. Crop Evol. 56: 323-330.

Guo HB, Li SM, Peng J and Ke WD (2007). Genetic diversity of Nelumbo accessions revealed by RAPD. Genet. Resour. Crop Evol. 54: 741-748.

Han YC, Teng CZ, Zhong S, Zhou MQ, et al. (2007). Genetic variation and clonal diversity in populations of Nelumbo nucifera (Nelumbonaceae) in central China detected by ISSR markers. Aquat. Bot. 86: 69-75.

Han YC, Teng CZ, Hu Z and Song YC (2008). An optimal method of DNA silver staining in polyacrylamide gels. Electrophoresis 29: 1355-1358.

Hu J, Pan L, Liu H, Wang S, et al. (2012). Comparative analysis of genetic diversity in sacred lotus (Nelumbo nucifera Gaertn.) using AFLP and SSR markers. Mol. Biol. Rep. 39: 3637-3647.

Jiang L, Wang L, Liu L, Zhu X, et al. (2012). Development and characterization of cDNA library based novel EST-SSR marker in radish (Raphanus sativus L.). Sci. Hortic. 140: 164-172.

Jung S, Abbott A, Jesudurai C, Tomkins J, et al. (2005). Frequency, type, distribution and annotation of simple sequence repeats in Rosaceae ESTs. Funct. Integr. Genomics 5: 136-143.

Kubo N, Hirai M, Kaneko A, Tanaka D, et al. (2009). Development and characterization of simple sequence repeat (SSR) markers in the water lotus (Nelumbo nucifera). Aquat. Bot. 90: 191-194.

Kumpatla SP and Mukhopadhyay S (2005). Mining and survey of simple sequence repeats in expressed sequence tags of dicotyledonous species. Genome 48: 985-998.

La Rota MR, Kantety V, Yu JK and Sorrels ME (2005). Nonrandom distribution and frequencies of genomic and ESTderived microsatellite markers in rice, wheat, and barley. BMC Genomics 6: 23.

Li Z, Liu XQ, Gituru RW, Juntawong N, et al. (2010). Genetic diversity and classification of Nelumbo germplasm of different origins by RAPD and ISSR analysis. Sci. Hortic. 125: 724-732.

Liu X, Qu W and Liang J (2010). Research progress of Nelumbo nucefera Gaertn. Strait Pharm. J. 3: 003.

Luro FL, Costantino G, Terol J, Argout X, et al. (2008). Transferability of the EST-SSRs developed on Nules clementine (Citrus clementina Hort ex Tan) to other Citrus species and their effectiveness for genetic mapping. BMC Genomics 9: 287.

Morgante M, Hanafey M and Powell W (2002). Microsatellites are preferentially associated with nonrepetitive DNA in plant genomes. Nat. Genet. 30: 194-200.

Ng P, Wei CL, Sung WK, Chiu KP, et al. (2005). Gene identification signature (GIS) analysis for transcriptome characterization and genome annotation. Nat. Methods 2: 105-111.

Nguyen Q (2001). Lotus for export to Asia: An agronomic and physiological study. Publication number 1/32, Rural Industries Research and Development Corporation, Barton, ACT 2600. 
Pan L, Xia Q, Quan Z, Liu H, et al. (2010). Development of novel EST-SSRs from sacred lotus (Nelumbo nucifera Gaertn) and their utilization for the genetic diversity analysis of N. nucifera. J. Hered. 101: 71-82.

Pan L, Quan Z, Hu J, Wang G, et al. (2011). Genetic diversity and differentiation of lotus (Nelumbo nucifera) accessions assessed by simple sequence repeats. Ann. Appl. Biol. 159: 428-441.

Parchman TL, Geist KS, Grahnen JA, Benkman CW, et al. (2010). Transcriptome sequencing in an ecologically important tree species: assembly, annotation, and marker discovery. BMC Genomics 11: 180.

Peng J and Lapitan NL (2005). Characterization of EST-derived microsatellites in the wheat genome and development of eSSR markers. Funct. Integr. Genomics 5: 80-96.

Rohlf F (2000). NTSYS-pc version $2.10 \mathrm{~m}$, Numerical taxonomy and multivariate analysis system, computer software, Exeter Software, New York.

Rozen S and Skaletsky H (2000). Primer3 on the WWW for general users and for biologist programmers. Methods Mol. Biol. 132: 365-386.

Sohn D-H, Kim Y-C, Oh S-H, Park E-J, et al. (2003). Hepatoprotective and free radical scavenging effects of Nelumbo nucifera. Phytomedicine 10: 165-169.

Thiel T, Michalek W, Varshney R and Graner A (2003). Exploiting EST databases for the development and characterization of gene-derived SSR-markers in barley (Hordeum vulgare L.). Theor. Appl. Genet. 106: 411-422.

Tian HL, Chen XQ, Wang JX, Xue JH, et al. (2008a). Development and characterization of microsatellite loci for lotus (Nelumbo nucifera). Conserv. Genet. 9: 1385-1388.

Tian HL, Xue JH, Wen J, Mitchell G, et al. (2008b). Genetic diversity and relationships of lotus Nelumbo cultivars based on allozyme and ISSR markers. Sci. Hortic. 116: 421-429.

Varshney RK, Graner A and Sorrells ME (2005). Genic microsatellite markers in plants: features and applications. Trends Biotechnol. 23: 48-55.

Wang Z, Fang B, Chen J, Zhang X, et al. (2010). De novo assembly and characterization of root transcriptome using Illumina paired-end sequencing and development of cSSR markers in sweetpotato (Ipomoea batatas). BMC Genomics 11: 726.

Wei LB, Zhang HY, Zheng YZ, Guo WZ, et al. (2008). Developing EST-derived microsatellites in sesame (Sesamum indicum L.). Acta Agron. Sin. 34: 2077-2084.

Xin Y, Cui H, Zhang M, Lin R, et al. (2005). Development of EST (expressed sequence tags) marker in Chinese cabbage and its transferability to repeseed. Hereditas 27: 410-416.

Xue J, Wang S and Zhou SL (2012). Polymorphic chloroplast microsatellite loci in Nelumbo (Nelumbonaceae). Am. J. Bot. 99: e240-e244.

Yang M, Han Y, Vanburen R, Ming R, et al. (2012a). Genetic linkage maps for Asian and American lotus constructed using novel SSR markers derived from the genome of sequenced cultivar. BMC Genomics 13: 653.

Yang, M, Han Y, Xu L, Zhao J, et al. (2012b). Comparative analysis of genetic diversity of lotus (Nelumbo) using SSR and SRAP markers. Sci. Hortic. 142: 185-195.

Zeng S, Xiao G, Guo J, Fei Z, et al. (2010). Development of a EST dataset and characterization of EST-SSRs in a traditional Chinese medicinal plant, Epimedium sagittatum (Sieb. Et Zucc.) Maxim. BMC Genomics 11: 94.

Zhang H, Wei L, Miao H, Zhang T, et al. (2012). Development and validation of genic-SSR markers in sesame by RNAseq. BMC Genomics 13: 316. 